\title{
An evaluation of the transition from BAME community mental health worker to IAPT low intensity psychological wellbeing practitioner
}

\author{
Naheem Hakim $^{1}$, Andrew R. Thompson ${ }^{2}$ and Gail Coleman-Oluwabusola ${ }^{3}$ \\ ${ }^{1}$ Sheffield Health and Social Care NHS Foundation Trust, UK \\ ${ }^{2}$ Department of Psychology, University of Sheffield, 1 Vicar Lane, Sheffield S1 2LT, UK \\ ${ }^{3}$ University of Sheffield and Independent Inquiry Into Child Sexual Abuse (IICSA), Home Office, UK
}

Received 7 April 2018; Accepted 21 November 2018

\begin{abstract}
The Improving Access to Psychological Therapies (IAPT) programme started in 2008, but it contained little provision for specifically meeting the needs of Black, Asian and minority ethnic (BAME) groups. The purpose of this evaluation was to describe the experience of transition from BAME community mental health worker (CMHW) to IAPT low-intensity psychological wellbeing practitioner (PWP) in order to identify possible gains and losses for the former communities served, and the factors that might contribute to successful training of people with BAME expertise. Four former CMHWs who had transitioned into working as PWPs were interviewed. Semi-structured interviews were used. The data were analysed using thematic analysis. Six major themes were identified with the benefits of training emerging as an important factor for the participants in enhancing their role. Three of the themes interconnected and focused on the impact for BAME communities in terms of access to service and barriers. Evident in the interviews were descriptions of adaptations that were made as a result of CMHW having access to both new and old skills. Finally, two themes focused on the participant recommendations as to how IAPT services might become more culturally responsive. The findings suggest that there can be significant benefits for services to provide IAPT training to people already providing culturally specific services. The participants reported that low-intensity cognitive behavioural therapy (LICBT) was effective, but only when cultural sensitive adaptations were made. The evaluation has some clear recommendations as to how IAPT services might seek to offer culturally responsive CBT. Suggestions for carrying out further practice-based evaluations are made.
\end{abstract}

Key words: BAME, cultural sensitivity, diversity, IAPT, low-intensity CBT, psychological training

\section{Introduction}

The Improving Access to Psychological Therapies (IAPT) programme started in 2008 to offer psychological therapies as recommended by the National Institute for Health and Care Excellence (NICE) guidelines. This took place after the publication of The Depression Report 
(Layard et al., 2006) highlighted that only one in four people were getting treatment for depression and anxiety in the UK. The report stated that people should be given a choice of psychological therapies. The IAPT service offers interventions based on the stepped care model (NICE, 2009). Initial interventions typically involve low-intensity cognitive behavioural therapy (LICBT), also known as guided self-help (Gellatly et al., 2007). The goal of LICBT is to provide evidence-based psychological interventions in a cost-effective fashion (Bennett-Levy et al., 2010). There is considerable evidence for the utility of this approach in treating common mental health problems (Clark et al., 2009; Webster et al., 2014).

In many European countries there are growing diverse populations and it is important that the mental health needs of these individuals are met, and that they have access to therapies and mental health techniques (Clark et al., 2009). It is well established that people from different cultures may have different understanding or features of depression (Papworth et al., 2013), and that some cultural factors may also be linked to resilience (Johnson et al., 2009).

The Delivering Race Equality in Mental Health Care (DRE) action plan, published in 2005, restated the need for mental health services to deliver equality and abolish discrimination for Black, Asian and minority ethnic (BAME) patients. One of the aims of this action plan was that by 2010 mental health services would be delivering appropriate services for BAME communities based on their mental health needs. However, despite this action plan there continues to be a clear need for more to be done within IAPT and other mental health services to meet the needs of BAME communities in removing barriers to receiving accessible and effective care (Papworth et al., 2013). The 2015 IAPT report indicated that a large number of staff are White British, with only $17 \%$ of the workforce being from a BAME background. Indeed, the Department of Health Positive Practice Guide (Department of Health, 2009) states that the IAPT services should be doing more to make services culturally responsive for BAME groups, and notably there is limited research on the effectiveness of CBT in patients of a Pakistani and Arab cultural background (Abudabbeh and Hays, 2006; Rahman et al., 2008; McIndoo and Hopko, 2014; Jameel and Ali, 2017).

In the evaluation of two pilot IAPT services after two years, it was found that individuals from BAME communities benefited from IAPT interventions (Clark et al., 2009). However, limited data were presented on patients' presenting problems and the definition of BAME was also limited with Asians of any background being categorized simply into one group. This is a significant flaw, as there is a lot of diversity between countries within the Asian continent (Mattern, 2002), which would suggest that the findings of this study are not readily generalizable to any specific ethnic group.

There have been studies demonstrating the effectiveness of CBT in a wide range of cultures and countries, but studies rarely describe the adaptations that were made to CBT in order for it to become culturally appropriate (Rahman et al., 2008). Indeed, there is acknowledgement that certain aspects of CBT might not sit easily within Arabic culture (Abudabbeh and Hays, 2006; McIndoo and Hopko, 2014). Abudabbeh and Hays (2006) in their review of the literature and drawing on their clinical experience criticized CBT for being focused towards Western patients based on the target for change typically being individual cognition and behaviour, which might fail to adequately consider the important role and value placed on systemic factors such as family and religion by patients of an Asian background. Another criticism has been levelled at 'cognitive restructuring' as it could potentially be misused to challenge beliefs that might be rooted in cultural or religious practices (Abudabbeh and Hays, 2006). 
In 2005, a community-based service, the Emotional Wellbeing Service, was established to provide support for Pakistani women and Yemeni men living in the North of Sheffield. The aim of this service was to reduce the stigma around mental health issues and for these communities to gain access to mental health services as they had previously been identified as not using the service available. Community mental health workers (CMHW) were specifically recruited to provide culturally appropriate support and these workers were provided with supervision by existing mental health services. In 2012, further funding was gained to expand the service to provide support for Pakistani men and Arabic speaking women.

An internal service evaluation conducted by A.R. Thompson and G. Coleman-Oluwabusola ('Evaluation of the Sheffield emotional wellbeing service', unpublished manuscript, 2014) found that this service was highly valued by service users and it was recognized that the CMHWs were able to encourage members of specific BAME communities to seek support, whereas formerly members of those communities had rarely accessed mental health services. However, it was also identified that the CMHWs lacked formal mental health training and had a desire to receive further training so as to enhance their skills. As a consequence, the evaluation report presented to the NHS commissioners recommended that the team be provided with places on the low-intensity IAPT training scheme run at the University of Sheffield, and that the CMHWs then consider how to adapt the LICBT techniques taught on the programme for use within their communities. The regional healthcare commissioners did not provide further funding for continuing the CMHW role as a standalone service, but sought to integrate the role via providing funding for the CMHWs to train as psychological wellbeing practitioners (PWPs). The purpose of the present study is to evaluate the experience of the CMHWs in receiving PWP training and of joining IAPT services.

Robinson et al. (2012) conducted a study examining role transition of mental health nurses to high-intensity CBT therapists. This is the only study to examine the experience of transition for mental health nurses to high-intensity CBT therapists. The findings of this small study $(n=6)$ revealed several factors that contributed to effective role transition and identified supervision as playing a crucial role. Whilst this study was small and used single interviews, it nevertheless provided important indicators for IAPT services in terms of providing guidance on service developments required in training so as to ensure successful role transitions in the provision of CBT.

To our knowledge there have not been any studies that have examined the transition of community mental health workers into PWPs or examined the use and adaptation of LICBT techniques by mental health workers with cultural expertise. Conducting such research is important, as there is recognition that CBT techniques may not be readily transferable for use with BAME service users without adaptation (Naeem et al., 2010). Additionally, identification as to how CMHWs with pre-existing cultural mental health expertise adapt LICBT may provide very useful and transferable information for CBT practitioners working with members of the BAME community.

The aim of this study was to look at the experience of transitioning from CMHW to IAPT PWP and the possible gains and losses of making this transition. This is relevant as these findings can help to consider what changes might be required to both IAPT services and to the delivery of LICBT so as to make them useful for BAME communities. Qualitative methods are particularly well suited to enabling detailed explorations of experience and processes such as role transition (Harper and Thompson, 2012), and as this study sought to gain detailed 
nuanced feedback, a descriptive form of thematic analysis (Joffe, 2012) was deemed to be the most appropriate method to use.

\section{Method}

\section{Participants}

For this study participants were selected (Emmel, 2013) on the basis of undergoing a transition from a CMHW to an IAPT PWP. Information sheets were sent to the potential participants informing them what the study was about and how they could participate. In total four participants volunteered, including one of the authors (N.H.). All participants had successfully completed a postgraduate training in low-intensity psychological interventions. Two of the participants had worked as CMHW for 1 year prior to undertaking the IAPT training, whereas the other two had been in the role for 7 and 6 years. The sample represents the total population available for study. Since completing IAPT training (approximately 3 years before), three of the participants had worked in the same area as they had when employed as CMHW, and one had been placed in another geographical area of the same city. The mean age of the group was 41.5 years $(S D=6.40)$ and consisted of two females (Asian British Pakistani and Yemeni) and two men (Asian British Pakistani and Yemeni).

\section{Data collection and analysis}

A semi-structured interview schedule was developed by the authors and modified following discussion with senior members of the IAPT management team, with regard to what they would like to ascertain in terms of service provision. A semi-structured interview technique was used, asking open-ended questions, giving the participants freedom to discuss issues they felt were relevant to their experience. Thus minimizing the possibility of participants being led by the interviewer and providing detailed information over other methods (McQueen and Knussen, 2006; Edwards and Holland, 2013; Silverman, 2013; Willing, 2013). The majority of the study questions were formulated by AT to minimise potential bias by $\mathrm{NH}$ in his dual role as Psychological Wellbeing Practitioner and researcher. The interviews took place on NHS or University premises. Interviews were conducted by NH (apart from the interview with NH which was conducted by A.R.T.). Participants completed consent forms. The interviews were recorded and were then transcribed by N.H. The data obtained were then analysed using thematic content analysis (TCA), which is used to find common themes in the data and to examine these in detail (Braun and Clarke, 2006; Joffe, 2012). This method was suited for the interview questions in this study exploring transition experiences.

Following Braun and Clarke's (2006) six steps, each transcript was read several times so as to familiarize with the data, noting any opinions or ideas. Three transcripts were analysed by N.H. and N.H.'s interview was analysed by G.C.-O. Then each line was read, making note of codes of interesting and relevant information. Codes were then put together into categories based on similarity, and themes and sub-themes were decided upon, based on the intuitive importance and to capture emerging patterns and meaning of the coded information in line with the aims of the study (Joffe, 2012). The themes were checked against the codes and then against the data, with the same method being used by N.H. and G.C.-O. N.H. and G.C.-O. then discussed the themes to ensure that there was inter-rater reliability between their analyses 
(Armstrong et al., 1997). Disagreements and differences in coding were resolved through discussion. There was minimal disagreement and the discussion led to an agreement being achieved on the final clustering of themes and on the thematic titles. The validity of the themes were checked by the use of member validation which involved sending the themes back to the participants for comment, and to ascertain that the results represented an appropriate reflection of their experience (Birt et al., 2016; Golafshani, 2003).

\section{Ethical approval}

Prior to clinical governance approval being gained from the NHS Trust, senior members of the service management team provided approval for the study to be conducted, and then ethical approval for the study protocol was gained from the ethics committee of the Department of Psychology, University of Sheffield.

\section{Results}

Pseudonyms are used for the quotes to maintain anonymity. Dots are used to show truncated quotes. Six major themes were discovered during the TCA, with each having a number of sub-themes.

\section{Training experience in two different roles}

The first major theme 'training experience in two different roles' has five major sub-themes: practice as an untrained Community Mental Health Worker, reflections on pre-IAPT training, useful training in IAPT, personal development in IAPT and a need for more training in IAPT to work with BAME patients. Participants shared their experience of having training in two different roles and how this was important for preparing them.

\section{Practice as an untrained Community Mental Health Worker}

Practice as an untrained Community Mental Health Worker was a common theme throughout the data for the participants when they shared their experience of working in the CMHS:

'... so with the previous role, there wasn't much training ... the IAPT manager came for one day $\ldots$ and that was just in the afternoon. That was the training that we got apart from the mandatory, NHS, training $\ldots$ but nothing to do with the role ... there was massive difference.' - Ahad

\section{Reflections on pre-IAPT training}

Not having enough training to prepare for the CMHS role led to the participants feeling overwhelmed and unsure about what they were supposed to be doing in their role as they were offering services such as counselling without having any training. This had a negative impact on their mental wellbeing and was possibly dangerous for patients:

'Hardly any training ... I remember offering counselling sessions to women, and ... one time I came up and I couldn't stop crying because it felt overwhelming to listen to people's problems, especially around sexual abuse, when they were younger and what happened to them, when you're 
Table 1. Major themes and sub-themes

\begin{tabular}{|c|c|}
\hline Major theme & Sub-themes \\
\hline $\begin{array}{l}1 \text { Training experience in } \\
\text { two different roles }\end{array}$ & $\begin{array}{l}\text { 1.1 Practice as an untrained CMHW } \\
\text { 1.2 Reflections on pre-IAPT training } \\
\text { 1.3 Useful training in IAPT } \\
\text { 1.4 Personal development in IAPT } \\
\text { 1.5 Need for more training in IAPT to work with BAME patients }\end{array}$ \\
\hline 2 Wider context & $\begin{array}{l}\text { 2.1 Flexible service for BAME patients } \\
\text { 2.2 Inaccessible IAPT } \\
\text { 2.3 Too structured } \\
\text { 2.4 BAME patients and suspicion of services }\end{array}$ \\
\hline 3 Differences encountered & $\begin{array}{l}\text { 3.1 Mental health understanding in different BAME communities } \\
\text { 3.2 Stigma } \\
\text { 3.3 Disengagement }\end{array}$ \\
\hline $\begin{array}{l}4 \text { Post-training adaptations } \\
\text { for BAME patients }\end{array}$ & $\begin{array}{l}\text { 4.1 Translating and modifying the material for BAME patients } \\
\text { 4.2 Overcoming barriers } \\
\text { 4.2 Adapting sessions and in between session tasks for patients } \\
\text { who cannot read or write }\end{array}$ \\
\hline $\begin{array}{l}5 \text { Undervalued cultural } \\
\text { and language expertise }\end{array}$ & $\begin{array}{l}\text { 5.1 Speaking another language } \\
\text { 5.2 More demanding role } \\
\text { 5.3 Helping colleagues } \\
\text { 5.4 Lack of recognition of expertise }\end{array}$ \\
\hline 6 Ideas for the future & $\begin{array}{l}\text { 6.1 Greeting patients in culturally appropriate ways } \\
\text { 6.2 Reaching out to isolated CMHS patients (remit) } \\
\text { 6.3 Tackle stigma } \\
\text { 6.4 Flexibility } \\
\text { 6.5 Promotion } \\
\text { 6.2 A new service and further necessary training }\end{array}$ \\
\hline
\end{tabular}

not trained, it hits you like a shock ... I didn't know how help them, how to support them, what I was meant to do. We were just doing things without being trained.' - Muna

\section{Useful training in IAPT}

However, their experience was different in the IAPT training, where they found it useful and felt it prepared them well to undertake their new role successfully. The importance of supervision was also highlighted:

'Out the training I think I learnt how to offer the PWP intervention, so the training was ... really good ... so I felt really well prepared ... and I was getting supervision ...' - Ahad

\section{Personal development in IAPT}

Participants shared how the IAPT training helped them with their personal development, such as self-confidence and gave them a sense of achievement. This experience led them to value training. However, they had not received this in their former role: 
'We didn't have any proper training in how to deliver talking therapies to people ... in wider community or BAME communities. So, I felt again a lot of knowledge, learning, confidence, selfesteem, has you know, boosted. I attended the university, it was a huge step.' - Muna

\title{
Need for more training in IAPT to work with BAME patients
}

All participants agreed that despite them valuing IAPT training they felt under-prepared for working with BAME patients, and their colleagues also felt the same. For example, colleagues would ask them for guidance on working with BAME patients. All participants agreed that more training was needed to successfully work with BAME patients, such as making adaptations. This is despite there being a module on diversity, which they felt was not sufficient:

'I could see when in discussions, in our team with team members that they said "oh, I'm working from, you know patient from BAME community, 'How did you do the session?', 'How long would you give them?' You know, 'which interpreters?' '. It needs further knowledge around how to adapt sessions to work with people from BAME communities.' - Muna

\section{Wider context}

The second major theme focused on the 'wider context' of both mental health services and their accessibility regarding BAME patients. This had four sub-themes: flexible services for BAME patients, inaccessible IAPT, too structured and BAME patients and suspicion of services

\section{Flexible service for BAME patients}

Sharing their experience of wider context, participants discussed how they felt the two services fared regarding offering services to BAME communities. In the former service, service flexibility and a wider remit for BAME patients was reported as an important factor in engagement:

\begin{abstract}
'It was a lot more flexibility. We weren't structured to say, only thing we do is an assessment and it's the first line intervention treatment plan, this is what we've got. With, that's what we got with IAPT with [the former service] if they were having court cases issues, where they were having divorce procedures, child access, stillborn children, where they were taking legal [action], court cases where they feel racially victimised, abused and everything. So, it supported all them areas where people feel they've just come to the country and they not getting the treatment and the respect that they should be getting ... but with the IAPT it's completely different structure, we are not counselling service with the PWPs, it's more about the treatment, and stepping them up for second, and third treatment [if necessary].' - Nigat
\end{abstract}

\section{Inaccessible IAPT}

All the participants shared their concern of how patients in their former service might be doing, as they were noted as not accessing the IAPT service. British Asians were also noted as not accessing IAPT. Importantly, IAPT only offers to see people in medical centres, whereas it was commented upon that the previous service had reached out to people, and provided a service in other venues as a way of increasing access: 
'The gap as I said, is to reach for those isolated people within their local communities ... you'll go to them [the community mental health worker]... [as they go to the] local community centres, to mosques. It's the word of mouth most of the times within the community.' - Ali

\section{Too structured}

The structure of the IAPT service compared with the former community service was another reason given by participants for BAME patients disengaging. It was suggested that the structure of the IAPT service placed limits as to what was on offer and what was not, therefore limiting what PWPs could offer the patients in a session:

'But with IAPT it is more assessment and treatments, it's more structured, it's not something that you can just say, if a patient walks in and says "right, I want to talk about my daughter, that's not getting married", and I can't sit there and listen to that, but with the [community service], sometimes it's [being] there as a hearing voice ...' - Nigat

'I think, just based on my experience working with the BAME communities, when I was working with the BAME community in the emotional wellbeing service they were attending more sessions, compared to what they are with the IAPT service. I think with the IAPT service they haven't, we haven't got that flexibility, like with the emotional wellbeing service it was tailored towards their needs basically, what they wanted and it was open.' - Ahad

\section{BAME patients and suspicion of services}

Participants shared that they thought that the information requirements of IAPT services represented a barrier for BAME patients to access services. As such, requests were often met with suspicion generally due to higher levels of stigmatization associated with feared repercussions should other people know that they had difficulties. The participant suggested that provision of information on services might help to reassure people:

'... you know women from BAME communities, it would help them to understand before referring them .... it's okay to talk to the GP, you're not going crazy, your kids aren't going to be taken away, if you told them [what to expect] it's a good thing [for] BAME communities.' - Muna

\section{Differences encountered}

The third major theme was the differences encountered with three sub-themes focusing on factors that were holding back BAME patients from engaging in the IAPT service. These sub-themes are: mental health understanding in different BAME communities, stigma and disengagement. PWPs used their experience in both roles to merge this knowledge and to subsequently address gaps in the service.

\section{Mental health understanding in different BAME communities}

Participants identified that different communities had different understanding of mental health. This was a common theme throughout the data and the participants had similar experiences with specific BAME communities. Within Pakistani communities the common themes reported by the participants were encountering service users as presenting their mental health somatically: 
'... they talk about aches, and feeling, you know, they think they are having a heart attack or they've got stroke, they having stroke, you know, they talk about the physical symptoms more, when they feeling stressed or anxious.' - Muna

In addition, some BAME communities such as Pakistani communities and African communities were reported as believing their mental health was a result of black magic:

'I know a few patients talked about, erm, black magic, just a few, where they said "I, you know, I started to feel down, I started to feel ill physically", have, you know they talked about when they described panic attacks, they said seizures but it wasn't seizures actually, it was a panic attack, when I explained this is a panic attack, they said erm, "I was fine so, I'm guessing this is black magic, somebody put, erm, what is it called, spell on me or something make me feel like this", just a few.' - Muna

From Muslim communities some individuals believed their mental health symptoms were due to Jinns (evil spirits). This could be misdiagnosed by experts who are not familiar with the culture:

'Okay ... for example the Muslim communities still believe in Jinn (spirit), for example if people say they have OCD ...' - Ali

Some individuals from Slovakian Roma communities believed their mental health symptoms were signs that dead relatives were trying to contact them. This came from their traditional beliefs:

'Their interpretation is it could be relatives ... people who have already passed away or something like that.' - Ali

\section{Stigma}

From their vast culture experience participants shared that stigma played an important role in patients not coming forward and accessing help as some individuals believed this will impact their family honour. This was the case for Pakistani and Arab communities, where individuals came and tried to hide their mental health problems:

'...they got the same symptoms, but they don't want to be diagnosed or have a label of mental health.' - Nigat

'Yeah, I mean, the, the women will come but they've not identified, they will try to hide the identity of mental health.'

'... so people are very reluctant in particular the patients and very reluctant to open up and talk about their personal situations about mental health because mental health is big stigma in the BME community and they don't want to be identified as having a mental health, so in front of the interpreters to talk about that they got this issue will be a bad thing.'

\section{Disengagement}

The issues described previously were cited as contributing to some BAME patients disengaging from treatment. In addition, disengagement was for some related to a lack of understanding of the potential benefits of psychological intervention based on a belief that medication was the only solution: 
'Err, where regards to some communities they just don't believe in it and they think it is just waste of time. To give you an example the Roma Slovak think, "that's not medication, we need medication" and it takes quite a lot of time for them to believe in it.' - Ali

\section{Post-training adaptations for BAME patients}

The fourth major theme was 'post training adaptations for BAME patients'. This had three sub-themes which focused on the adaptations culture and language expert PWPs made for the service to be culturally responsive for BAME patients. These sub-themes are: translating and modifying the material for BAME patients, overcoming barriers, adapting sessions and in between session tasks for patients who cannot read or write.

Participants made IAPT services responsive to BAME communities by not using technical language, making the cases studies culturally appropriate, normalizing their condition by discussing the prevalence of mental health issues, modifying the material, including family and religion into five areas, CBT model, supporting patients to build their faith, focusing on family rather than just the individual and translating the material.

\section{Translating and modifying the material for BAME patients}

'There, there was some changes, so for example when we talked about cognitive restructuring, we didn't say cognitive restructuring, we talked about unhelpful thinking and how we challenge and balance this. We didn't go to deep into the treatment interventions, for example behavioural activation, we didn't say behavioural activation we said, you know, we talked about doing more to help you to feel better and the value flower, we talked about this, the values that people ... and which areas they feel they can link themselves more to. But we tailored, yes some of the content of the, you know.' - Muna

'... for example the case studies, we changed them to, for example Fatima and Muhammad, Fatima a housewife and Muhammad a taxi driver. And we thinking of another case study where Muhammad is, for example working for council or working for in a factory and his wife is doing a part time job because Muhammad and Fatima, taxi driver and a housewife doesn't represent everybody in the BME community.' - Muna

'... I shared my five area, areas that I've translated in Arabic and also the cognitive restructuring forms, were translated as well.' - Ali

\section{Overcoming barriers}

Participants faced a number of barriers and through experience learnt how to overcome these; they shared how knowing the language and the culture helped them to identify what the barriers were. This helped them to engage patients in the treatments:

'You would include for example case studies ... about the different people from different communities ... For example, to understand their needs, improve communication ... like I said maybe tailoring some material making it simple, not for all of them, some of them are sharp and they understand it very quick, but some cannot read or write in their own languages and they have no clue what you're talking about, so, if you stick, stick to the model and they say, that they are not going to engage and they will not benefit from it and they will become worse and just leave and disengage. These type of skills are so vital and crucial for all PWPs.' - Muna 
'... also religion came in as well, so when I was seeing a lot patients, first they were saying to me, "only God can help me, I am suffering from depression and this is from God, so only God can take my depression away, you can't do anything." I helped the patient to understand how they got this condition .... and how the treatment works, and also in Islam it says you have to help yourself, before God can help you (from a Hadith: 2517 narrated by Anas Bin Malik: 2517 as cited in Muflinhun (n.d.): "O Messenger of Allah! Shall I tie it and rely (upon Allah), or leave it loose and rely (upon Allah)?" He said: "Tie it and rely (upon Allah)." So just knowing about Islam was important, so I was able to challenge them with the things that they were saying were based on religion.' -Ahad

\section{Adapting sessions and in between session tasks for patients who cannot read or write}

Drawings, pictures and voice recordings (mobile) were used for patients who could not read or write to track their daily activities and report these back in the sessions. This was done in a sensitive way so as not embarrass the patients by giving them all the options of tracking their progress and letting them choose. Here is an example of overcoming barriers:

'... when I was working with someone, err, because she couldn't read or write, err, I just thought it will be easier for her to use pictures instead, to show, you know, what she was doing, and, err, smiley face and sad face to rate her mood.' - Ahad

\section{Feedback}

Participants shared their experience of positive feedback from patients who had received a culturally responsive service. In this example Nigat challenged a patient who believed her only purpose was to look after her husband and children, which is a norm in certain cultures. Nigat highlighted to the patient why it was important that she took care of herself, as if she got worse she would struggle to be productive. Nigat also helped her to understand she was not being selfish or a bad wife if she cared for herself more. She highlighted the benefits to the patient. The patient's feedback was:

'... you know what, you were right, you were absolutely right, what you said, you know I've done this and it has really benefited me, and, err, I'm, I'm going out more often, my husband's noticed a difference in my mood, my children have noticed me less irritable and I was always doing routine and necessary activities all the time, you know for my children and my husband and you know but I forgot myself and now I have realised that (.), err, I need to bit more for this.' - Nigat

\section{Undervalued cultural and language expertise}

The fifth major theme 'undervalued cultural and language expertise' focused on expertise that the PWPs in this evaluation have and the lack of recognition of their influence not only patients but mental health staff. This has four sub-themes: speaking another language, more demanding role, helping colleagues and lack of recognition of expertise.

\section{Speaking another language}

The participants shared how their language skills were important, especially when it came to working with patients who can only speak a specific language. This removed the three-way conversation with interpreters, which was problematic for some patients due to confidentiality: 
'... I've seen that patients who come from for example Yemen or Syria, or Iraq, they, they could relate more to therapist who speaks their language, they can be themselves, you know, and having an interpreter three way, you, ... we can't do without it but it's more ideal when someone speaks your language. They can feel where you come with the language, the words that they use, how they suffer ... they have that connection with you.' - Muna

\section{More demanding role}

Participants shared that they felt that they had found the PWP role demanding as they had had to make additional time so as to make adaptations to the standard interventions available:

'However, the skills that we are developing are very exhausting ... we're translating the material in another language. It's taking me longer to type the notes up because they're in second language ...' - Nigat

The participants also shared that they believed their cultural expertise and the lack of availability of similar expertise elsewhere in the service, meant that they sometimes ended up working with presenting problems not usually addressed within the psychological wellbeing role:

'[we sometimes] drift into step three.'

\section{Helping colleagues}

Participants discussed how they tried to manage the demand by sharing the translated material and giving colleagues in their team, advice. They also gave advice to other health professionals and gave examples, showing how important culture understanding and sensitivity is:

'They had a woman who they sectioned, because she was throwing water everywhere, I actually went in to see her ... It was OCD, she was doing wudu (Islamic procedure for washing parts of the body, is required before praying). And her, she wasn't throwing water, she was washing her arms and washing her feet in the sink, because she kept on purifying herself ... I explained to the staff ... she is not deliberately throwing water.' - Nigat

"Patients had tried step two intervention but did not engage, so when I saw them they were saying the reason they had not engaged was because suggestions made to them were not culturally appropriate, so, like with BA, they were being told for pleasurable activities, go out spend time with your friends, go to the gym, doing the things outside the home, that will be pleasurable. But from my own cultural experience, I knew that for some women it makes them feel uncomfortable going out with their friends to have a meal, so it was just making changes, based from my knowledge about culture ...' - Ahad

\section{Recognition of expertise}

As previously stated whilst the participants shared how they believed that they worked particularly hard so as to facilitate and engage BAME patients into the IAPT service, they expressed that they sometimes felt that they did not receive the recognition for this input:

'... we are really cramped into do all the work ... and in additional another language, but we are not actually being recognised...' - Nigat 


\section{Ideas for the future}

The final major theme coming out of analysis was 'ideas for the future' and had six subthemes: greeting patients in culturally appropriate ways, reaching out to isolated CMHS patients (remit), tackle stigma, flexibility, promotion and a new service and further necessary training. Participants shared the adjustments IAPT service should make in the training courses to offer culturally relevant service for BAME patients.

\section{Greeting patients in culturally appropriate way}

Greeting patients in a culturally appropriate way was discussed as being very important for breaking the barriers and building rapport. Colleagues in the IAPT service who were advised to do this, found it helpful in engaging with their BAME patients. One PWP shared her experience:

'Culturally adapted, and, and you will say as-salaam-alaikum (peace be upon you), if they say, you know, as-salaam-alaikum or they leaving you have to say wa-alaikum-assalam (and on you be peace), you can't say, “oh, good bye”, or, you know, you being ignorant, you know, you're telling them this is a wall between me and you. It doesn't work you have to say where's that, erm, culturally adapted and, and we feel, make them feel comfortable.' - Muna

'... you respected them basically ... sometimes if it's female patients and it's a male worker, too much eye contact might make her feel uncomfortable, especially if she's not educated and she's shy or isolated person.' - Muna

\section{A new service and further necessary training}

With regard to the remaining recommendations, participants felt one way to make IAPT more efficient for the BAME patients as well as the staff is to have a specialist mini IAPT team, which works with all the BAME patients from the city rather than being restricted to one area. They also suggested that there was a clear need to have people with cultural and language expertise available in all 'steps' of the IAPT pathway:

'We still drift to certain extent to providing CBT at step three, therefore, I think we need some sort of training.' - Ali

\section{Discussion}

To our knowledge this is the first evaluation looking at staff transition from a specialist BAME community worker role to an IAPT psychological wellbeing practitioner role. The findings indicate that transitioning community mental health workers from BAME-specific services into mainstream mental health services has the potential to assist in meeting the call for mental health services to better meet the mental health needs of the whole population (Delivering Race Equality in Mental Health Care: DRE, 2005). As such there is clearly a need to consider other types of community worker making the transition into IAPT services and for this transition to be carefully evaluated so as to ensure that BAME communities benefit from such developments and attempts to integrate services. Existing IAPT services are largely based on a Western model of understanding psychological distress and of providing treatment, and in order for IAPT services to better serve BAME communities, such services must actively 
encourage people with a range of backgrounds to join mental health services, and to make use of their cultural knowledge so as to enhance service provision.

Six major themes emerged from the analysis of the interviews of role transition from community mental health worker to psychological wellbeing practitioner. Benefiting from training and the requirement for further training came up as important factors for the participants. The next three themes were interconnected and focused on the BAME patients: accessing the service, the barriers they faced and adaptations that were made for them. The final two themes focused on the participants' recommendations as to how IAPT services might become further culturally responsive.

The findings from the first theme are supported by previous studies looking at CBT training, where participants felt more competent in delivering CBT after receiving comprehensive training (Bennett-Levy and Beedie, 2007). Training also helped participants transition into their new role, as was found in a study by Robinson et al. (2012), where support by supervisors in training was highlighted as an important factor.

The participants reported that the training received when they had worked as community mental health workers had been insufficient, and contributed to them having felt overwhelmed. This was also possibly dangerous for patients as they were not receiving evidence-based help and was a key driver in the recommendation of the initial service evaluation that the CMHWs be with access to psychological wellbeing training. In addition, some service users attending the community service disclosed abusive experiences. The participants were clear that since having joined the IAPT service they were not better able to manage risk.

Participants in this evaluation reported having success in working with BAME patients, but they reflected on the need for modifying LICBT techniques to better engage service users. Rathod et al. (2010) also found in their research, that CBT is an appropriate treatment for severe mental health problems if adapted culturally and flexibly for BAME patients.

It is well documented that people from different cultures have different understanding of concepts like depression (Papworth et al., 2013). The participants in this study reflected on how their confidence grew in addressing some of these issues once they had completed training. However, it was not simply about receiving training on mental health but also on drawing on their wider cultural and religious knowledge. For example, one participant shared how they were able to challenge a patients' beliefs about jinns by drawing on knowledge of both the Quran and the evidence base for causes of common mental health problems; these findings are supported by Islam and Campbell (2014).

Stigma around mental health is not just experienced by BAME communities but is often more likely to be associated with fear of damaging family honour or marriage opportunities than in other communities (Al-Darmaki and Sayed, 2004). Shame and family honour were factors which Pilkington et al. (2012) found were a strong indicator whether British Asians accessed psychological help. The participants gave examples as to how they were able to manage some of these concerns so as to encourage people to use IAPT services. The management of such concerns required not only cultural awareness and sensitivity but also knowledge of LICBT techniques that might be adapted in such a way as so to not increase the risk of creating further feelings of shame. The participants recognized the role that Mosques and local Imams might also play in reducing stigma, and described how their local knowledge and links were useful in reassuring patients that the service they were receiving was appropriate.

Participants made adaptations using their experience and knowledge of barriers for specific communities. They structured their sessions following LICBT guidelines (Richards and 
Whyte, 2011), but translated, and modified the material. They ensured that the pace was right, so patients did not have difficulties understanding them as guided self-help is intense and requires the therapist to guide and support the patient in a way where they fully understand the therapist (Coull and Morris, 2011). They gave extra time to patients who could not read or write. Drawings, pictures and voice recordings (using a mobile phone) were used for them to track their daily activities and report these back in the sessions. There was also consideration of the family and religion rather than sole focus on the self, which is recommended in the literature for when working with BAME patients (Tseng et al., 2005; Abudabbeh and Hays, 2006).

The former CMHWs who had joined IAPT services are only available in one part of the city and there remains a potential gap in the provision of culturally sensitive services elsewhere in the region, and the participants reported how they had been providing informal consultation to their colleagues, who have been able to make successful adaptations to their delivery of LICBT. Ghassemzadeh (2007) stated that it is important for clinicians to share their experiences of working with different communities as this helps with making adaptations in CBT for each community. The participants in this study made recommendations as to how their adaptations to LICBT might be delivered during PWP training. The participants shared that adaptations were not enough on their own and the use of language and cultural awareness is important, and they felt that this cultural knowledge was a valuable asset that they held and wished to share. For example, Abudabbeh and Hays (2006) recommend that therapists be responsive to patients' cultural norms, such as accepting a hug, as not doing so could be misinterpreted as being disrespectful. However, in IAPT the policy is not to hug the patients due to having clear boundaries. Broad policies that cover exchanges between care workers and service users might benefit from being reviewed by service users from a range of backgrounds so that they might be revised to be sufficiently flexible to reduce the risk of offending people from a BAME background.

This evaluation has several methodological limitations such as the small sample size and the inclusion of one of the authors as a participant. However, the sample size reflected the entire sample available and we believe the results may have some transferability to other similar services. In addition, to the small sample size, it is important to acknowledge that the participants knew the interviewer as a colleague, and an independent interviewer might have gained differing accounts from the participants. Participants were also asked to recall a transition that had occurred approximately 5 to 6 years ago, so there may well have been some memory bias. Finally, the study would have been enhanced if it had included some feedback on the effectiveness and satisfaction of the interventions provided by the participants.

The findings of this study indicate the potential value in purposively selecting staff providing specific services to BAME populations to receive training in the delivery of LICBT interventions, and to then join mainstream mental health services. The study clearly emphasizes how transition into main stream mental health services for such mental health workers can enhance service provision in very specific and useful ways beyond simply providing one-to-one support to service users. For example, the participants in this study actively commented on how their cultural knowledge and reflections as to how to adapt LICBT was in demand from colleagues from across the service. However, there is also an indication that services need to continue to monitor and reflect upon how to make the best use of cultural expertise of such workers beyond the beyond the completion of training and the initial transition period. 


\section{Main points}

(1) The IAPT programme started in 2008, but it contained little provision for specifically meeting the needs of Black, Asian and minority ethnic (BAME) groups.

(2) This evaluation explored the experience of transition of four PWPs from BAME community mental health worker (CMHW) to IAPT low-intensity PWP to identify possible gains and losses for the former communities served, and the factors that might contribute to successful training of people with BAME expertise.

(3) There were a number of findings which suggest that there can be significant benefits for services to provide IAPT training to people already providing culturally specific services.

(4) The participants reported that low-intensity CBT (LICBT) was effective, but only when culturally sensitive adaptations were made.

(5) The evaluation has made some clear recommendations as to how IAPT services might seek to offer culturally responsive CBT.

(6) We acknowledge the limitations of this evaluation and recognize the need for collection of data from BAME patients accessing the service.

\section{Acknowledgements}

We would like to thank the Psychological Wellbeing Practitioners who participated in this evaluation.

\section{Conflicts of interest}

The authors of this paper have no conflicts of interest with respect to this publication.

\section{Ethical statement}

Ethical approval was obtained from senior members of the service management team, clinical governance from the NHS Trust and ethics committee of the Department of Psychology, University of Sheffield.

\section{Financial support}

This evaluation was unfunded.

\section{Recommended follow-up reading}

Abudabbeh N, Hays PA (2006). Cognitive-behavioral therapy with people of Arab heritage. In PA Hays and GY Iwamasa (eds), Culturally Responsive Cognitive-Behavioral Therapy: Assessment, Practice, and Supervision (pp. 141-159). Washington, DC, USA: American Psychological Association.

Islam F, Campbell RA (2014). 'Satan has afflicted me!' Jinn-possession and mental illness in the Qur' an. Journal of Religion and Health 53, 229-243.

Naeem F, Gobbi M, Ayub M, Kingdon D (2010). Psychologists experience of cognitive behaviour therapy in a developing country: a qualitative study from Pakistan. International Journal of Mental Health Systems 4, 2. 


\section{Reference}

Abudabbeh N, Hays PA (2006). Cognitive-behavioral therapy with people of Arab heritage. In PA Hays and GY Iwamasa (eds), Culturally Responsive Cognitive-Behavioral Therapy: Assessment, Practice, and Supervision (pp. 141-159). Washington, DC, USA: American Psychological Association.

Al-Darmaki F, Sayed M (2004, August). Practising polymorphism in traditional gender-role Emirates: conflicts and challenges. In CZ Enns (Chair), Learning from international psychologists about feminist and social justice issues. Symposium conducted at the 112th annual meeting of the American Psychological Association, Honolulu, HI.

Armstrong D, Gosling A, Weinman J, Marteau T (1997). The place of inter-rater reliability in qualitative research: an empirical study. Sociology 31, 597-606.

Bennett-Levy J, Beedie A (2007). The ups and downs of cognitive therapy training: what happens to trainees' perception of their competence during a cognitive therapy training course? Behavioural and Cognitive Psychotherapy 35, 61-75.

Bennett-Levy J, Richards DA, Farrand P, Christensen H, Griffiths KM, Kavanagh DJ, Proudfoot J (2010). Low intensity CBT interventions: a revolution in mental health care. Oxford Guide to Low Intensity CBT Interventions, 3-18.

Birt L, Scott S, Cavers D, Campbell C, Walter F (2016). Member checking: a tool to enhance trustworthiness or merely a nod to validation? Qualitative Health Research 26, 1802-1811.

Braun V, Clarke V (2006). Using thematic analysis in psychology. Qualitative Research in Psychology 3, 77-101.

Clark DM, Layard R, Smithies R, Richards DA, Suckling R, Wright B (2009). Improving Access to Psychological Therapy: initial evaluation of two UK demonstration sites. Behaviour Research and Therapy 47, 910-920.

Coull G, Morris PG (2011). The clinical effectiveness of CBT-based guided self-help interventions for anxiety and depressive disorders: a systematic review. Psychological Medicine 41, 22392252.

Department of Health (2009). Positive Practice Guide. Available at: https://www.uea.ac.uk/ documents/246046/11919343/black-and-minority-ethnic-BAME-positive-practice-guide.pdf (accessed 28 January 2018).

Edwards R, Holland J (2013). What is Qualitative Interviewing? London: Bloomsbury.

Emmel N (2013). Sampling and Choosing Cases in Qualitative Research: A Realist Approach. London: Sage.

Gellatly J, Bower P, Hennessy S, Richards D, Gilbody S, Lovell K (2007). What makes selfhelp interventions effective in the management of depressive symptoms? Meta-analysis and metaregression. Psychological Medicine 37, 1217-1228.

Ghassemzadeh H (2007). The practice of cognitive-behavior therapy in Roozbeh Hospital: some cultural and clinical implications of psychological treatment in Iran. American Journal of Psychotherapy 61, 53.

Golafshani N (2003). Understanding reliability and validity in qualitative research. The Qualitative Report 8, 597-606.

Harper D, Thompson A (2012). Qualitative Research Methods in Mental Health and Psychotherapy: A Guide for Students and Practitioners. Oxford: John Wiley and Sons.

Islam F, Campbell RA (2014). 'Satan has afflicted me!' Jinn-possession and mental illness in the Qur'an. Journal of Religion and Health 53, 229-243.

Jameel B, Ali S (2017). The effectiveness of low intensity cognitive behavioral therapy (LI-CBT) on reducing symptoms of depression in Arab clients.

Joffe H (2012). Thematic analysis. Qualitative Research Methods in Mental Health and Psychotherapy: A Guide for Students and Practitioners 1, 210-223. 
Johnson H, Thompson AR, Downs M (2009). Non-Western interpreters' experiences of trauma: culturally protective factors following exposure to extreme stress. Ethnicity and Health 14, 407-418.

Layard R, Bell S, Clark DM, Knapp M, Knapp M, Meacher M, Priebe S (2006). The Depression Report: A New Deal for Anxiety and Depression Disorders. London: London School of Economics. Available at: http://cep.lse.ac.uk/pubs/download/special/depressionreport.pdf

Mattern J (2002). Asia: World's Largest Continent. New York: The Rosen Publishing Group, Inc.

McIndoo CC, Hopko DR (2014). Cognitive-behavioral therapy for an Arab college student with social phobia and depression. Clinical Case Studies 13, 128-145.

McQueen RA, Knussen C (2006). Introduction to Research Methods and Statistics in Psychology. Harlow: Pearson Education Ltd.

Muflinhun (n.d.). Jami` at-Tirmidhi Book 35 Hadith 2517. Available at: https://muflihun.com/ tirmidhi/35/2517 (accessed 28 January 2018).

Naeem F, Gobbi M, Ayub M, Kingdon D (2010). Psychologists experience of cognitive behaviour therapy in a developing country: a qualitative study from Pakistan. International Journal of Mental Health Systems 4, 2.

NICE (2009). Depression in adults: the treatment and management of depression in adults. Clinical guideline CG90. National Institute for Clinical Excellence: London.

Papworth M, Marrinan T, Martin B, Keegan D, Chaddock A (2013). Low Intensity Cognitive Behaviour Therapy: A Practitioner's Guide. London: Sage Publications Ltd.

Pilkington A, Msetfi RM, Watson R (2012). Factors affecting intention to access psychological services amongst British Muslims of South Asian origin. Mental Health, Religion and Culture 15, $1-22$.

Rahman A, Malik A, Sikander S, Roberts C, Creed F (2008). Cognitive behaviour therapy-based intervention by community health workers for mothers with depression and their infants in rural Pakistan: a cluster-randomised controlled trial. The Lancet 372, 902-909.

Rathod S, Kingdon D, Phiri P, Gobbi M (2010). Developing culturally sensitive cognitive behaviour therapy for psychosis for ethnic minority patients by exploration and incorporation of service users' and health professionals' viCMHT and opinions. Behavioural and Cognitive Psychotherapy 38, 511533.

Richards D, Whyte M (2011). Reach Out: national programme student materials to support the delivery of training for psychological wellbeing practitioners delivering low intensity interventions (3rd edn). London: Rethink Mental Illness.

Robinson S, Kellett S, King I, Keating V (2012). Role transition from mental health nurse to IAPT high intensity psychological therapist. Behavioural and Cognitive Psychotherapy 40, 351366.

Silverman D (2013). Doing Qualitative Research: A Practical Handbook. London: Sage.

Tseng WS, Chang SC, Nishizono M (2005). Asian Culture and Psychotherapy. Honolulu: University of Hawaii Press.

Willing C (2013). Introducing Qualitative Research in Psychology. Maidenhead: Open University Press.

Webster R, Thompson AR, Webb TL, Sheeran P (2014). Self-help treatments and stepped care. In P Emmelkamp and T Ehring (eds), The Wiley Handbook of Anxiety Disorders (pp. 1242-1255). London: Wiley. 


\section{Learning objectives}

(1) To learn about factors contributing to successful clinical role transitions.

(2) To understand the complexity of working therapeutically with patients from ethnic minority backgrounds.

(3) To consider useful insights into Slovakian Roma community use of mental health services.

(4) To describe successful adaptations for BAME patients receiving low-intensity cognitive behavioural therapy.

(5) To describe strategies for managing stigma associated with mental health from an Islamic perspective. 